\title{
WATER BODY EXTRACTION FROM MULTI SPECTRAL IMAGE BY SPECTRAL PATTERN ANALYSIS
}

\author{
Nguyen Dinh Duong \\ Department of Environmental Information Study and Analysis, Institute of Geography, \\ 18 Hoang Quoc Viet Rd., Cau Giay, Hanoi, Vietnam - duong.nguyen2007@gmail.com
}

Commission VIII, WG VIII/4

KEY WORDS: Remote sensing, Hydrology, Classification, Automation, Spectral pattern

\begin{abstract}
:
Water is one of the vital components of the Earth environment which needs to be frequently monitored. Satellite multispectral remote sensing image has been used over decades for water body extraction. Methodology of water body extraction can be summarized to three groups: feature extraction, supervised and unsupervised classification and data fusion. These methods, however, are of pure mathematical and statistical approach and little of them explore essential characteristics of multispectral image which is based on ground object radiance absorption behaviour in each sensing spectral bands. The spectral absorption characteristics of water body in visible and infrared bands differ very much from the other ground objects. They depend only on the used spectral bands and can be considered as invariant and sensor independent. In this paper the author proposed an application of spectral pattern analysis for water body extraction using spectral bands green, red, near infrared NIR and short wave infrared SWIR. The proposed algorithm has been used for water body extraction by Spot 5 and Landsat 5 TM images. Ground truth validation was carried out in Hanoi City. The advantage of this algorithm does not base on water body extraction only but it allows to asses also water quality. Different level of turbidity and organic matter contents could be classified by using additional index.
\end{abstract}

\section{INTRODUCTION}

Due to the importance for the Earth environment water has been monitored for a long time. Recently remote sensing approach in water recognition has been intensively studied and satellite remote sensing image data has been recognized as very useful information source for water extraction. Methodologies of water body extraction can be summarized to three groups: feature extraction, supervised and unsupervised classification and data fusion. Rajiv Kumar Nath et al. (2010) provided comprehensive overview on methods on water extraction from high resolution satellite images. Fu June et al. (2007) developed an automatic extraction of water body from TM image using decision tree algorithm. The proposed algorithm is based on spectral characteristics of water body in TM images. From the 6 visible bands author selected four bands $b_{2}, b_{3}, b_{4}$ and $b_{5}$ for development of decision tree algorithm which allows automatically water extraction. The algorithm is based on reflectance threshold for band $b_{4}$, relation between $b_{4}, b_{5}$ and $b_{2}$ and $b_{5}$ and relation between sum of $b_{2}, b_{3}$ and $b_{4}, b_{5}$. Validation was done by visual checking of analysis and manual interpretation. Hua Wang and others (2008) developed water extraction method based on texture analysis. The algorithm works for high resolution panchromatic imagery. Spectral characteristics of water were not used in the proposed algorithm. Jiancheng Luo et al. (2010) presented very interesting approach in water extraction using Landsat TM images. The algorithm combines water index computation, whole-scale segmentation, whole-scale classification and localscale segmentation and classification to achieve high-precise water extraction result. More complicated algorithm has been proposed by Min Li et al (2011) for water body extraction based on oscillatory network. Panu Nuangjumnong (2009) used Spot pan-sharpened image for water extraction. There are many other methods for water extraction which can be named in long list. In this paper the author proposed quite original algorithm for water extraction. Nguyen Dinh Duong (1997) proposed a method for decomposition of multi-spectral image into several sub-images based on modulation (spectral pattern) of the spectral reflectance curve. The hypothesis roots from the fact that different ground objects have different spectral reflectance and absorption characteristics which are stable for a given sensor. This spectral pattern can be considered as invariant and be used as one of classification rules. Water body is commonly understood as collection of water on the Earth surface. They are ocean, lakes, rivers, ponds and others geographical features. It is very easy to recognize water body in conventional survey practice. Remote sensing, however, observes the Earth surface in entire field of view of sensor and records unselectively ground objects. Water recorded in remote sensing images can be not only lakes, rivers, ponds, reservoir but also rice field after irrigation and others features. In this paper the author does not differentiate the true water body from temporal water surface.

\section{METHODOLOGY}

\subsection{Spectral Pattern of Water body}

Given a dataset with four spectral bands: green, red, near infrared and short wave infrared $\mathbf{X}\left(\mathrm{b}_{1}, \mathrm{~b}_{2}, \mathrm{~b}_{3}, \mathrm{~b}_{4}\right)$ where the $\mathrm{b}_{\mathrm{i}}$ stands for reflectance in band i converted from digital number DN of each pixel using the gain and bias coefficients provided along with image data. A spectral reflectance curve can be constructed by simple plotting of reflectance values in a graph as showed on Figure 1. If we use the $C$ for value of relative positions of each vertex then modulation or spectral pattern of the spectral reflectance curve could be expressed as: $\mathrm{C}_{12} \mathrm{C}_{13} \mathrm{C}_{14} \mathrm{C}_{23} \mathrm{C}_{24} \mathrm{C}_{34}$. The $\mathrm{C}$ value is of $\{0,1,2\}$. For example 
for pixel vector $(130,100,70,76)$ the code will be 222220 . Because the reflectance of clear water is high in green band, low in red and near infrared and almost absorbed in short wave infrared band therefore it is always $b_{1}>b_{3}, b_{1}>b_{4}, b_{2}>b_{3}, b_{2}>b_{4}$, so the $\mathrm{C}_{13}, \mathrm{C}_{14}, \mathrm{C}_{23}, \mathrm{C}_{24}$ are always 2 . For turbid water it can happen that reflectance in red band due to sedimentation is higher than reflectance in green band. Based on experiments the author found that water body is involved mainly in pattern 222222, 022222, and 002222. Modulation of spectral reflectance curve itself is not enough for water extraction because there are many spectral mixtures that have the same spectral reflectance as water. To separate water from possible mixtures the ratio of green / SWIR, red /SWIR or reflectance value of the SWIR could be used depending on type of water: clear or turbid (organic matter contaminated).

\subsection{Data Used for Experiments}

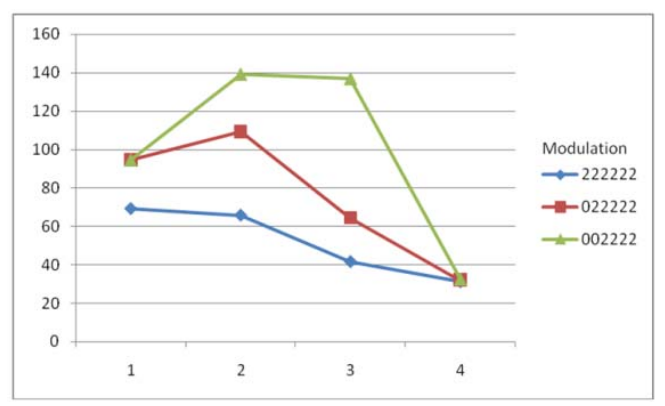

Figure 1. Three main modulations of spectral reflectance curve for water body
To demonstrate automatic water body extraction the Landsat TM scene 127-45 observed on 27/12/1993 and Spot 5 scene 269-307 observed on 24/06/2003 have been used as input data. Image windows of Hanoi area have been cut from full scenes for study. The calibration coefficients gain and bias for conversion from DN to reflectance are given in the metadata files along with the image data. Only 4 bands of Landsat 5 TM image have been used. The bands 2, 3, 4 and 5 of Landsat 5 TM and bands 1, 2, 3 and 4 of Spot 5 HRG have almost the same spectral sensitivity as showed in Table 1.

\begin{tabular}{|c|c|c|c|}
\hline \multicolumn{2}{|c|}{ Landsat 5 TM sensor } & \multicolumn{2}{c|}{ SPOT 5 HRG sensor } \\
\hline Band number & $\begin{array}{c}\text { Sensitivity } \\
(\mu \mathrm{m})\end{array}$ & Band number & $\begin{array}{c}\text { Sensitivity } \\
(\mu \mathrm{m})\end{array}$ \\
\hline 1 & $0.45-052$ & N/A & N/A \\
\hline 2 & $0.52-0.6$ & 1 & $0,50-0,59$ \\
\hline 3 & $0.63-0.69$ & 2 & $0,61-0,68$ \\
\hline 4 & $0.76-0.9$ & 3 & $0,79-0,89$ \\
\hline 5 & $1.55-1.75$ & 4 & $1,58-1,75$ \\
\hline 6 & $10.4-12.5$ & N/A & N/A \\
\hline 7 & $2.08-2.35$ & N/A & N/A \\
\hline
\end{tabular}

Table 1. Spectral characteristics of Landsat 5 TM and SPOT 5 HRG

Figure 2 displays windows of Landsat 5 TM and Spot 5 HRG respectively for study area. Colour composition is red=SWIR, green=NIR, blue=red. The nominal spectral sensitivities of both sensors TM and HRG are almost the same but in practice we can observe differences in image data.
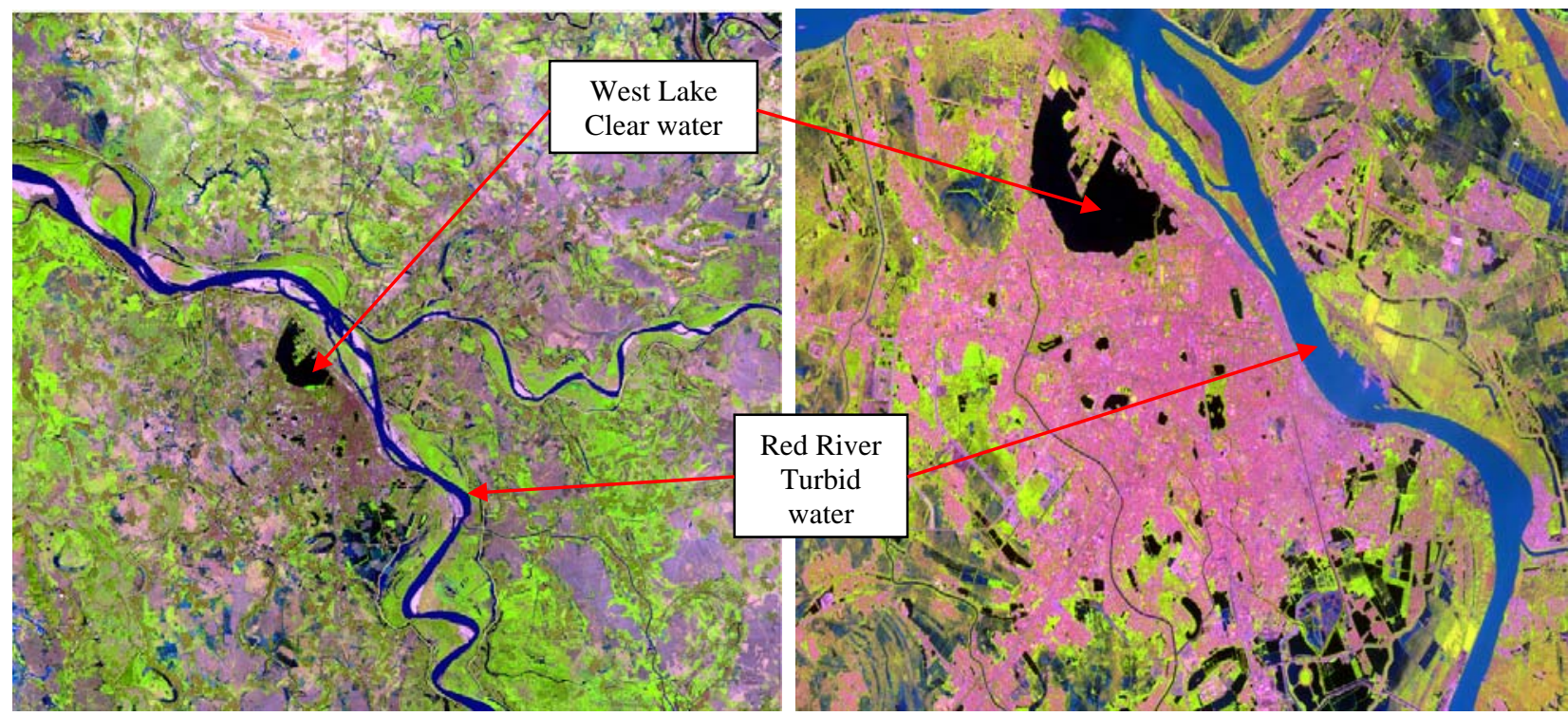

Figure 2. Study area by Landsat 5 TM (left) and Spot 5 (right)

Pixels which have spectral reflectance curve with modulation 222222 are showed on Figure 3. In this modulation while the Spot 5 image shows almost only clear water bodies like lakes and ponds in the study area, the Landsat 5 TM image display beside clear water also many other ground objects which are mostly infrastructure and construction. The largest water body in these images is the West Lake which is natural lake in Hanoi. On the TM image the mixture of urban and construction appears in dark purple colour. We can see few pixels along the Red River in blue colour. The Spot 5 image in this spectral pattern displays more correctly clear water bodies. We do not see almost any mixture at all.

The pixels with modulation 022222 for Spot 5 image show some ponds and lakes together with mixtures of construction objects. However the Landsat 5 TM image tends higher mixture rate when comparing to Spot 5 data. Modulation 022222 of Landsat 5 TM shows turbid water, sandy surface, irrigated paddy field and also construction.

Though the spectral sensitivity of both sensors of Landsat 5 TM and Spot 5 HRG is almost the same for 4 bands from green to SWIR but image data created by them are quite different in 
implementation. These differences are caused maybe by different spatial resolution, sensor construction or viewing angle of the sensor during observation. While water body is captured in Landsat 5 TM only in modulation 222222 and 022222, for Spot 5 HRG, water is located in 4 modulations: 222222, 022222, 002222 and 002022.

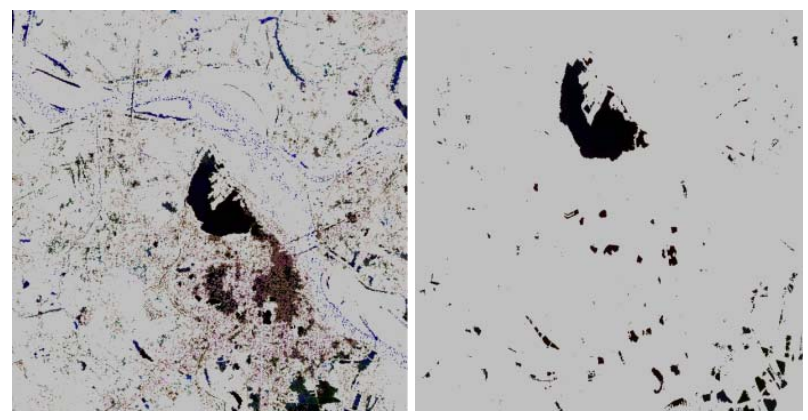

Figure 3. Water by modulation 222222 for Landsat 5 TM (left) and Spot 5 (right)

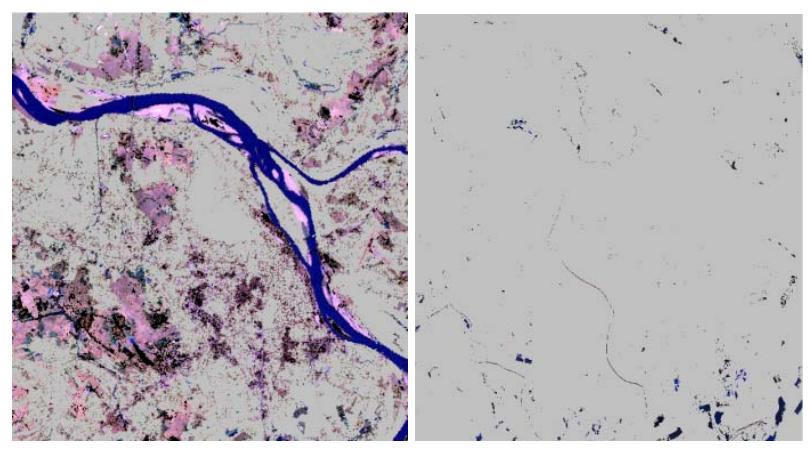

Figure 4. Modulation 022222 for Landsat 5 TM (left) and Spot 5 (right)

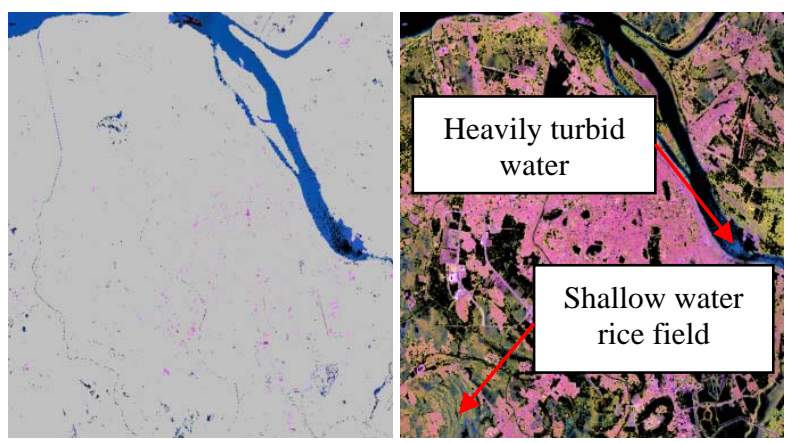

Figure 5. Modulation 00222 (left) and 002022 (right) for Spot 5

For Spot 5 HRG clear water is captured mainly in modulation 222222 and 022222 and turbid water has reflectance curve modulation as 002222 and 002022 . In modulation 002022 there are heavily turbid water and very shallow water rice field (Figure 5).

\subsection{Water Body Extraction Algorithm}

For new comers in spectral pattern analysis there is almost unknown knowledge about how many spectral patterns for the given image could be and what ground objects a particular spectral pattern stand for. The author developed an useful utility to decompose the given image into sub-images containing only pixels of single spectral pattern. Statistics on how many spectral patterns the given images can be decomposed and number of pixels for each spectral pattern are given in Table 2. Blue colour filled rows indicate spectral patterns for water bodies.

\begin{tabular}{|c|c|c|c|}
\hline \multicolumn{2}{|c|}{ Landsat 5 TM image } & \multicolumn{2}{c|}{ Spot 5 HRG image } \\
\hline $\begin{array}{c}\text { Spectral } \\
\text { pattern }\end{array}$ & $\begin{array}{c}\text { Number of } \\
\text { pixels }\end{array}$ & $\begin{array}{c}\text { Spectral } \\
\text { pattern }\end{array}$ & $\begin{array}{c}\text { Number of } \\
\text { pixels }\end{array}$ \\
\hline 022222 & 848613 & 002022 & 1568859 \\
\hline 202022 & 787863 & 202022 & 394708 \\
\hline 222222 & 240233 & 002222 & 154754 \\
\hline 002022 & 239842 & 222222 & 86738 \\
\hline 002222 & 90760 & 022222 & 40344 \\
\hline 222022 & 42689 & 222022 & 4597 \\
\hline
\end{tabular}

Table. 2 Statistics on spectral patterns and number of pixels for each spectral pattern for Landsat 5 TM and Spot 5 HRG image of study area. Colour shaded rows indicate spectral patterns for water.

Some sub images with single spectral pattern have been showed in Figure 2, 3, 4 and 5. After confirmation which spectral pattern contains water surface the analysis was implemented by the following algorithm. The water body extraction is consisted of 4 steps for Landsat TM and 6 steps for Spot 5 HRG:

a. Reading image data and finding out gain and bias coefficients for conversion from DN to reflectance.

b. Conversion from DN to reflectance and determining modulation of spectral reflectance curve for each pixel vector

c. Case 222222: applying different threshold values for SWIR band of TM and HRG sensors to extract water.

d. Case 022222: applying different threshold values for SWNIR band of TM and HRG sensors to extract water.

e. Case 002222 - Spot 5 only: applying threshold value for SWNIR band to extract water.

f. Case 002022 - Spot 5 only: applying threshold value for SWIR band to extract water.

It is obvious that the algorithm is composed of two parts: decomposition of the image into sub images according to spectral reflectance patterns and level slicing into water and land using different threshold values for the SWIR band. If we apply a single threshold value for water extraction there might be under or over estimation in clear and turbid water area because reflectance of turbid water is always higher than clear water. Figure 7 explains water body under estimation by level slicing of Spot SWIR band.

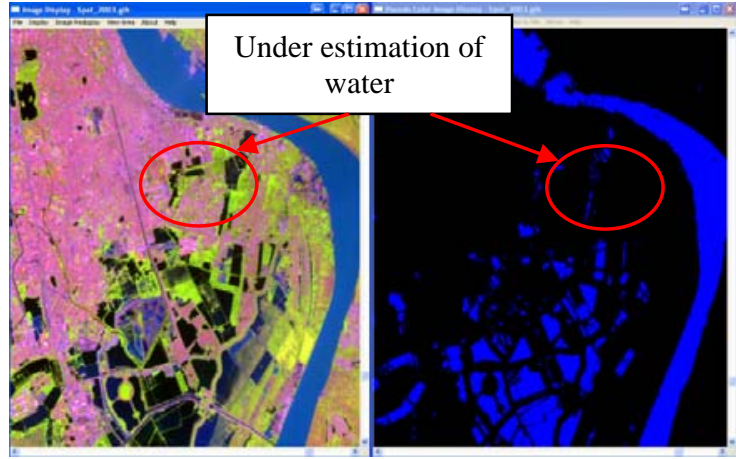

Figure 6. Spot 5 colour composite image (left) and water extraction by level slicing of SWIR band (right) 


\section{RESULT AND DISCUSSION}

By interactive visual checking a set of threshold values for Landsat 5 TM and Spot 5 HRG were established. The Table 3 shows the threshold values of band SWIR for all spectral patterns.

\begin{tabular}{|c|c|c|}
\hline Spectral patterns & Landsat 5 TM & Spot 5 HRG \\
\hline 022222 & 3.0 & 45.0 \\
\hline 222222 & 3.5 & 90.0 \\
\hline 002222 & N/A & 40.0 \\
\hline 002022 & N/A & 37.0 \\
\hline
\end{tabular}

Table 3. Threshold values for spectral patterns in Landsat 5 TM and Spot 5 HRG sensors
Based on the proposed algorithm and chosen threshold values water extraction for the Landsat 5 TM and Spot 5 HRG had been carried out. Figure 7 shows result of water body extraction of Landsat 5 TM image and Figure 8 displays Spot 5 HRG water body extraction. Water body filled by solid red colour over 4, 3, 2 colour composites is showed on right sites in both figures. The study area on Land sat 5 TM image is larger than on the Spot 5 HRG. The study area includes Hanoi City and surrounding area. Main water bodies are lakes, ponds, rivers and canals. In colour composite SWIR=red, NIR=green and Green=blue vegetation appears as green, urban including housing, construction appear in purple colour. Clear water is showed in dark blue and turbid water in cyan colour with different level of brightness depending on sedimentation contamination grades.

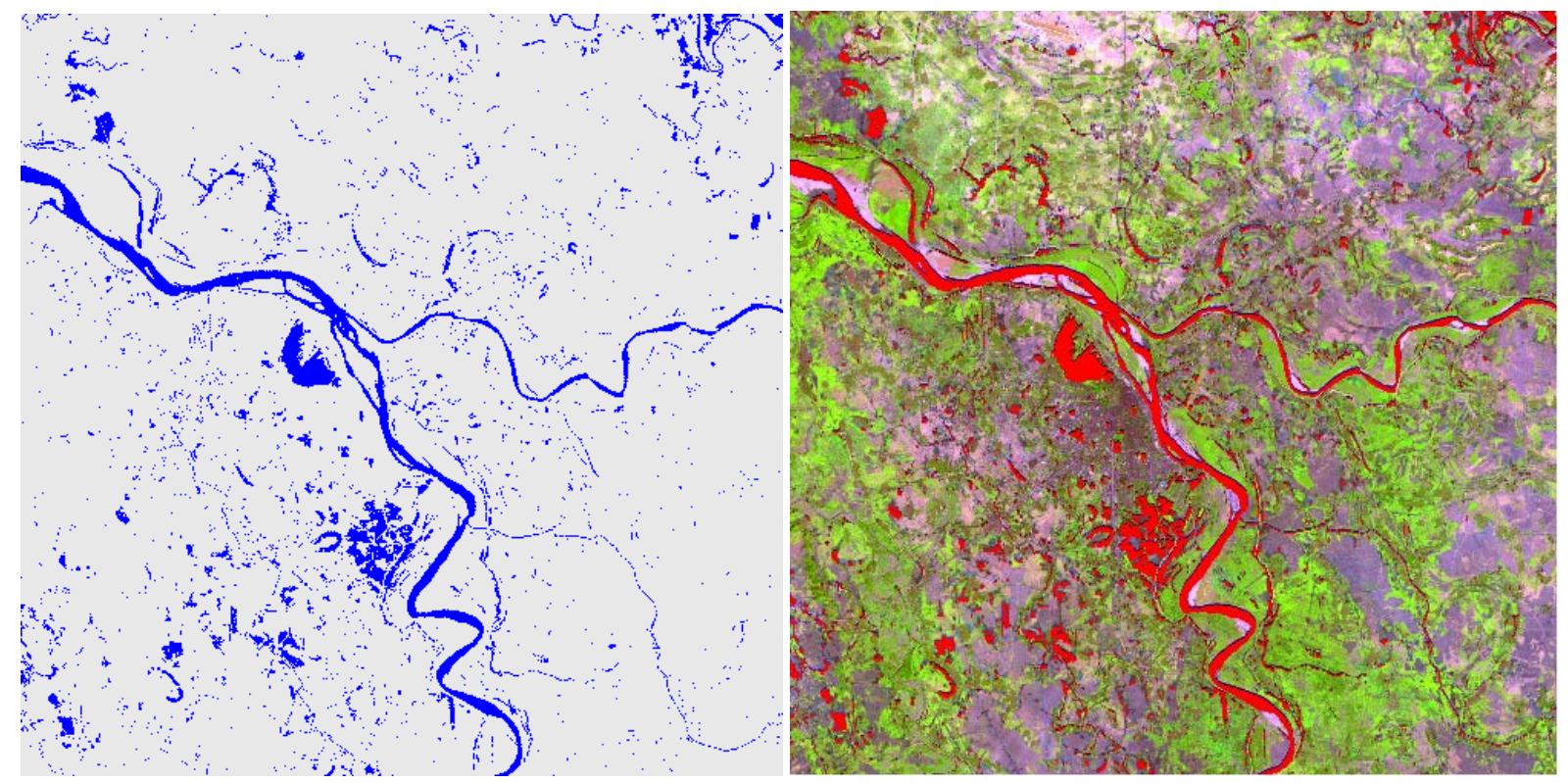

Figure 7. Water extraction by Landsat 5 TM image (left) and overlay on colour composite (right)

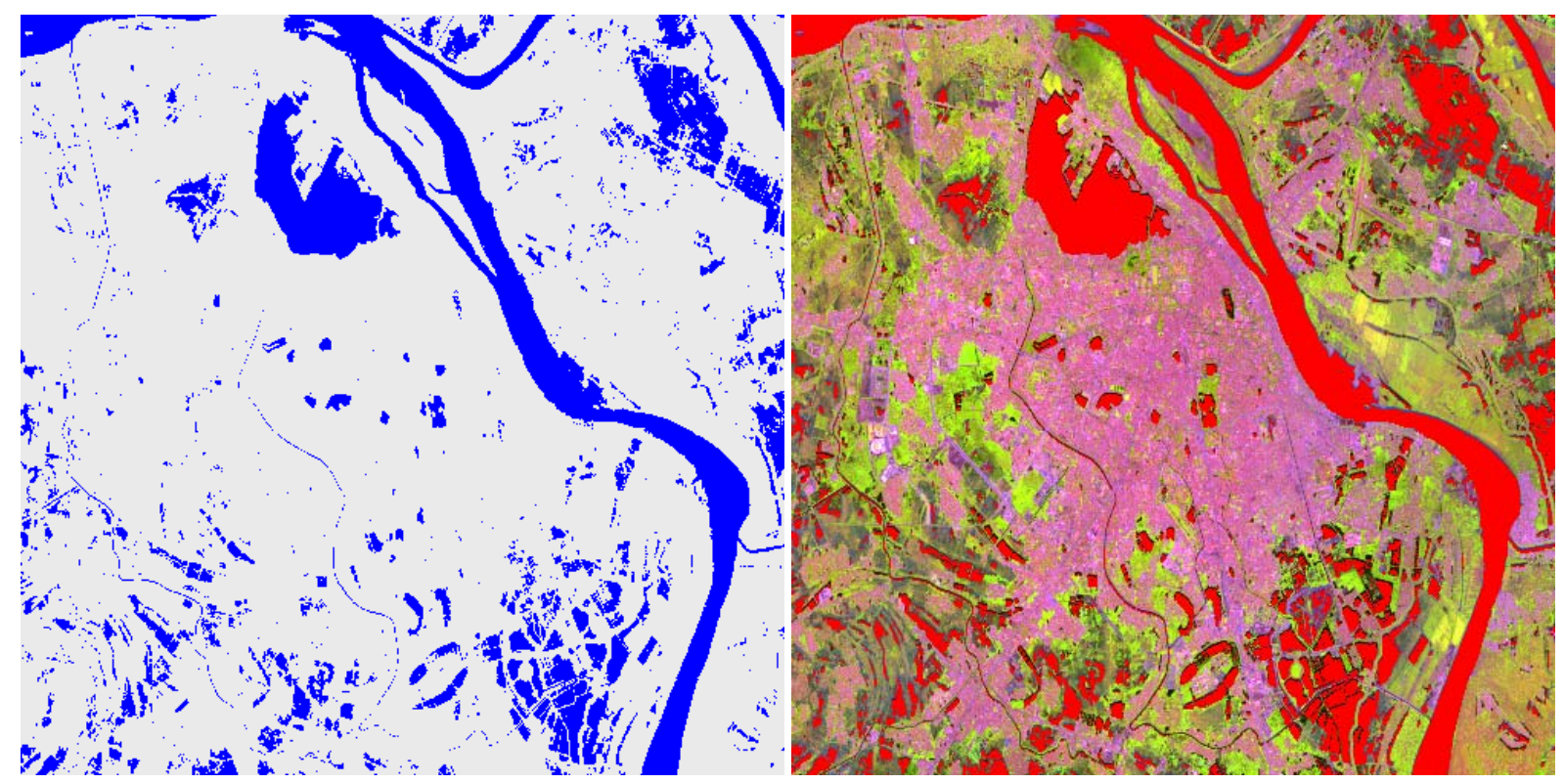

Figure 8. Water extraction by Spot 5 HRV image (left) and overlay on colour composite (right) 
To assess accuracy of the water extraction a comparison of water extraction result and input image by visual check has been made. Figure 9 shows enlargement of West Lake and part of Red River on Landsat 5 TM. Water extraction is showed on top and colour composite of input image on bottom part. We can see that boundaries of main water bodies with large area are drawn quite exactly. Some small canals are not fully extracted due to low spatial resolution of Landsat 5 TM sensor.

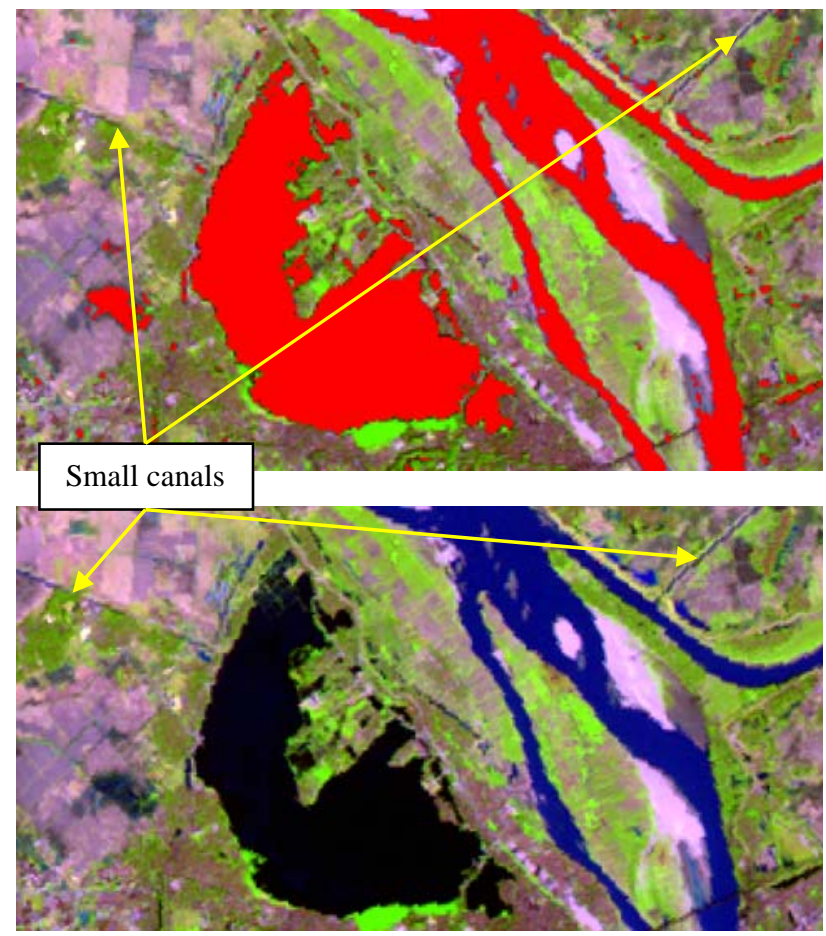

Figure 9. Water body extraction for Landsat 5 TM (top),) and in colour composite (bottom)

Figure 10 shows comparison study for Spot 5 HRG image. All water bodies which are widely enough, at least 5 pixels were very exactly extracted. Small water area is mixed with surrounding ground objects and cannot be extracted.
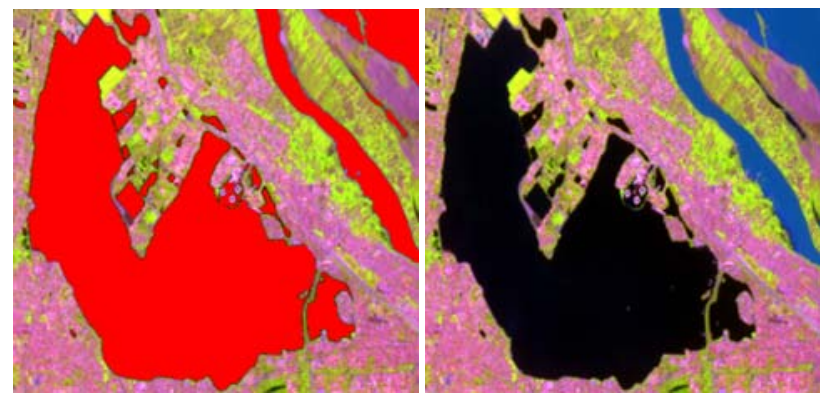

Figure 10. Water body extraction for Spot 5 HRG (top), ) and in colour composite (bottom)

Due to $10 \mathrm{~m}$ spatial resolution of the Spot 5 HRG sensor, many spectral mixtures occur in the image. The mixture allocated mainly in the boundary of water body. Therefore when we enlarge enough the result we can see thin unclassified boundary around extracted water body. Normally due to low spatial resolution the Landsat $5 \mathrm{TM}$ image is suitable for mapping in scale 1:100,000 and Spot 5 HRG image for scale 1:50,000. The mapping standard requires accuracy of $0.3 \mathrm{~mm}$ in map scale for boundary of all hydrographical features. This requirement is easily fulfilled for large area water feature but quite difficult for long thin water features. Figure 11 explains in detail this phenomenon.

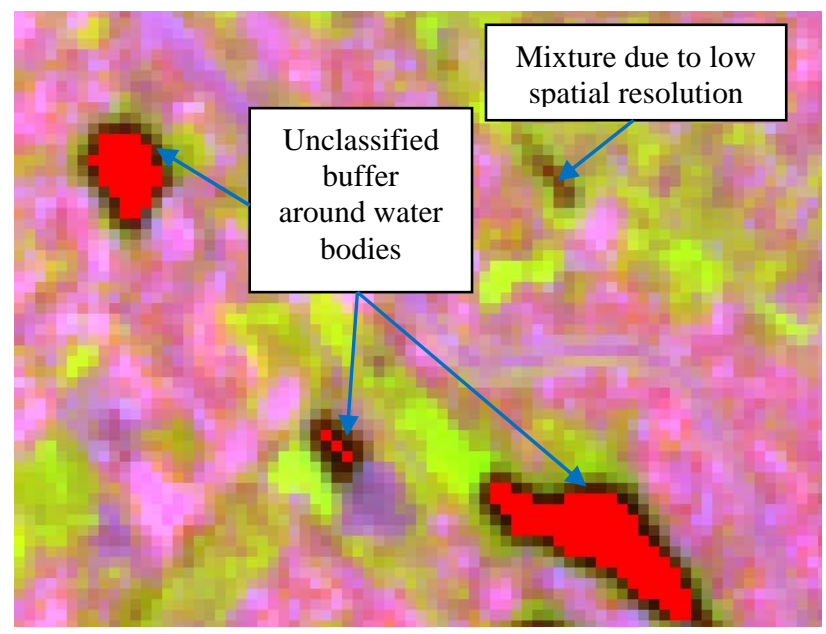

Figure 11. Influence of spatial resolution to accuracy of water extraction

\section{CONCLUSION}

The study pointed out some main conclusions as follow:

- The spectral pattern of water in four spectral bands: green, red, NIR and SWIR can be used for water extraction.

- Due to spectral mixture of ground objects caused by low spatial resolution water extraction by spectral patterns needs to be combined with some other measures such as level of reflectance in SWIR band.

- $\quad$ Even there are unclassified pixels around water body or water body which is narrower than 5 pixels in width cannot be classified but when we consider mapping scale 1:100,000 for Landsat $5 \mathrm{TM}$ and 1:50,000 for Spot 5 HRG sensors the accuracy achieved by this method can be without doubt fairly accepted.

- $\quad$ In the next phase the author would like to extend research for Landsat TM and ETM+ data with all 6 visible bands.

\section{References:}

Nguyen Dinh Duong, 1997. Graphical analysis of spectral reflectance curve. Proc. of ACRS 1997.

Fu June, Wang Jizhou, Li Jiren, 2007. Study on the automatic extraction of water body from TM image using decision tree algorithm. Proc. of SPIE Vol. 6625 662502-1.

Hua Wang, Li Pan, Hong Zheng, 2008. Multi-texture-model for water extraction based on remote sensing image. Proc. of 2008 Congress on Image and Signal processing. IEEE computer society.

Luo, J., Sheng, Y., Shen, Z., Li, J., 2010. High-precise water extraction based on spectral-spatial coupled remote sensing information. In IGARSS(2010) 2840-2843.

Rajiv Kumar Nath, S K Deb, 2010. Water-body Area extraction form high resolution satellite images - an introduction, review, 
International Archives of the Photogrammetry, Remote Sensing and Spatial Information Sciences, Volume XXXIX-B8, 2012 XXII ISPRS Congress, 25 August - 01 September 2012, Melbourne, Australia

and comparison. International Journal of Image Processing (IJIP), Volume (3): Issue (6).

Min Li, Lizhong Xu, Min Tang, 2011. An Extraction of water body of remote sensing image based on oscillatory network. Journal of Multimedia, Vol. 6, No. 3. 